\title{
Die Entwicklung von Joint Ventures in Rußland
}

\author{
Georgij Bagijev / Alexander Parfenov*
}

Joint Ventures und Tochterbetriebe stellen eine höchst anziehende Form für Auslandskapitalanlagen in Rußland dar. Statistische Daten bestätigen dies: Die Zahl der Betriebe mit Auslandskapitaleinlagen hat sich in Rußland von 1990 bis 1994 mehr als versechsfacht (vgl. Tab. 1) und betrug am 01.01.1995 13300 Unternehmen; ihr Gesamtkapital beläuft sich auf 2,3 Billionen Rubel; der Auslandskapitalgeberanteil daran beträgt 8,7\%, der der russischen Partner 41,3\%, (die übrigen Anteile hält der Staat). Die Analyse der Branchenstruktur dieser Unternehmen in der Volkswirtschaft Rußlands zeigt, daß der größte Teil sich auf die Bereiche Handel und öffentliche Versorgung (38\%) sowie die Industrie (28\%) konzentriert. Die absoluten Zahlen sind: ${ }^{5}$

$\begin{array}{ll}\text { Handel und Dienstleistungen } & 5049 \text { Unternehmen } \\ \text { Industrie } & 3698 \\ \text { Bauwesen } & 811 \\ \text { Transport und Kommunikation } & 389 \\ \text { Logistikbetriebe } & 242 \\ \text { Landwirtschaft } & 120 \\ \text { Forstwirtschaft } & 58 \\ \text { Fischerei } & 56 \\ \text { andere Betriebe } & 539\end{array}$

Im Bereich Infrastruktur ist der größte Teil der Betriebe mit Auslandskapital (657) auf dem Gebiet der Wirtschaftsbeziehungen tätig, außerdem arbeiten 237 Unternehmen im Gesundheitswesen und Sozialfürsorgebereich, 196 Unternehmen auf dem Gebiet Kredite/Finanzen/Versicherung, 141 im

Georgij Bagijev, geb. 1941, Prof. Dr., Leiter Marketing Abteilung, St. Petersburger Universität der Ökonomie und Finanzen, aktuelle Arbeitsgebiete: Ökonomie und Theorie, Methodologie und Methoden verschiedener Marketingsysteme und kommerzieller Kommunikation, Theorie von Marktnetzwerken, Benchmarking.

Alexander Parfenov, geb. 1962, Dr. Ph., Assistenzprofessor an der Handelsfakultät, St. Petersburger Universität der Ökonomie und Finanzen, aktuelle Arbeitsgebiete: Entwicklung von Joint Ventures und ausländischen Wirtschaftstätigkeiten in der Russischen Föderation, Methoden der Handelslogistik, ökonomische Mechanismen des Handelsaustausches.

5 Lebedev, W.: Die Kapitaleinfuhr: Die Ziffern sind sehr groß, aber die Summe ist zu klein. In: Die Wirtschaft und das Leben. Nr. 50. Bd. 1. 1995. (russ.) 
Kulturbereich, 115 Unternehmen auf dem Ausbildungssektor. Im gesamten Infrastrukturbereich beträgt der Anteil der Unternehmen mit ausländischen Kapitaleinlagen etwas über $10 \%$.

Das größte Interesse gilt, unserer Meinung nach, der Verteilung der Joint Ventures und der Auslandsunternehmen, die im Industriebereich tätig sind: Von den 3698 Industriebetrieben mit Auslandsinvestitionen arbeiten 1072 Unternehmen (29\%) im Maschinenbau- und Metallbearbeitungsbereich, 844 Betriebe (23\%) in der Holz-, Zellstoff- und Papierindustrie, 455 Unternehmen (12\%) in der Nahrungsmittelindustrie, 393 Betriebe (11\%) in der Leichtindustrie, 149 Unternehmen (4\%) in der chemischen und erölverarbeitenden Industrie, 785 Betriebe (21\%) in anderen Industriebereichen. Es sei vermerkt, daß am 01.01.1995 die Zahl der Betriebe mit Auslandskapitaleinlagen, die wirklich mit der Arbeit begonnen haben, 9887 betrug (74,3\% der Gesamtzahl angemeldeter Unternehmen).

Tabelle 1: Grundkennziffern der Tätigkeit der Unternehmen mit Auslandskapitaleinlagen in Rußland

\begin{tabular}{|l|c|c|c|c|c|}
\hline & 1990 & 1991 & 1992 & $1993 *$ & 1994 \\
\hline $\begin{array}{l}\text { Anzahl der angemeldeten Unternehmen } \\
\text { mit Auslandseinlagen (zum Jahresende) }\end{array}$ & 1971 & 2022 & 2533 & $\begin{array}{c}\text { etwa } \\
9000\end{array}$ & 13300 \\
\hline $\begin{array}{l}\text { Umfang der Produktions-, Arbeits- und } \\
\text { Dienstleistungsherstellung (realen } \\
\text { Preisen) in Milliarden Rubel }\end{array}$ & 3,2 & 18,4 & 449 & - & 12900 \\
\hline $\begin{array}{l}\text { Zahl der Beschäftigten (zum Jahresende) } \\
\text { in Tausend }\end{array}$ & 66 & 137 & 195 & 273 & 331 \\
\hline $\begin{array}{l}\text { Ausfuhr (in realen Außenhandelspreisen) } \\
\text { in Mio. US\$ }\end{array}$ & 325 & 727 & 1863 & 2640 & 4700 \\
\hline Einfuhr (in realen Preisen) in Mio. US\$ & 1276 & 810 & 2037 & 3056 & 3600 \\
\hline $\begin{array}{l}\text { Produktionsleistung auf dem Binnen- } \\
\text { markt } \\
\text { in Milliarden Rubel } \\
\text { in Mio. US\$ }\end{array}$ & $\begin{array}{c}2,5 \\
\text { * alle Daten 1993 vom Ende der ersten Halbjahres }\end{array}$ & $\begin{array}{c}11,25 \\
568\end{array}$ & 1365 & 521 & 11500 \\
\hline
\end{tabular}

Tabelle 1 zeigt, daß während des betrachteten Zeitraums auf dem Gebiet des Gemeinschafts- und Auslandsgewerbes in Rußland die wesentlichsten Veränderungen passiert sind: Die Anzahl der Betriebe mit Auslandskapitaleinlagen hat sich versechsfacht, die Zahl der Beschäftigten hat sich verfünffacht, die Produktionsleistung (auch der Arbeit und Dienstleistungen) wuchs um den Faktor 14,5 an, die Einfuhr wurde verdreifacht. Im Endergebnis kann man sagen, daß diese Unternehmen einen positiven Außenhandelssaldo (1,1 Mrd. US\$) aufweisen. Dies zeigt deutlich die Zunahme der Bedeutung dieses Wirtschaftsbereiches für die Entwicklung der russischen Volkswirtschaft. 
Außerdem wächst die Anzahl der Staaten, deren natürliche und juristische Personen an der Schaffung von Joint Ventures und Auslandsunternehmen in Rußland teilnehmen. Im Jahre 1990 waren es noch Vertreter aus 51 Staaten, 1993 waren es bereits 120 Staaten, im Jahre 1994 deren 128. Aus folgenden Ländern stammen die meisten Partner: USA 1908 Unternehmen (14,3\% der Gesamtzahl); Deutschland 1517 (11,4\%); China 1019 (7,7\%); Finnland 791 (5,9\%); Großbritannien 666 (5\%); Italien 563 (4,2\%); Polen 497 (3,7\%); Österreich 466 (3,5\%); Schweiz 368 (2,8\%); Bulgarien 350 (2,6\%); Japan 301 (2,3\%); Frankreich 301 (2,3\%); Schweden 300 (2,2\%); Lettland 271 (2,0\%); Türkei 244 (1,8\%). Die angeführten Daten zeigen, daß die Vertreter von 15 Staaten rund $72 \%$ der Gesamtanzahl an Unternehmen mit Auslandskapital angemeldet haben.

In der letzten Zeit wächst auch die Zahl von Joint Ventures und Auslandsunternehmen, die mit der Beteiligung von Partnern aus Osteuropa gebildet werden. Im Endeffekt existierten 1994 in Rußland 497 Betriebe mit Kapital aus Polen; 350 Unternehmen mit Kapital aus Bulgarien; 212 Unternehmen mit Kapitaleinlagen aus Ungarn; 237 aus der ehemaligen Tschechoslowakei, 186 aus dem ehemaligen Jugoslawien. Der Grund für diese Zunahme besteht darin, daß deren West-Orientierung sich nicht bewährt hat und sie deshalb um den Wiederaufbau der früher existierenden Wirtschaftskontakte bemüht sind.

Die Regionalforschung zeigt, daß der größte Teil der Betriebe mit Auslandsinvestitionen sich auf die folgenden Regionen konzentriert: Moskau 5597 Unternehmen (42\% der Gesamtanzahl); St. Petersburg 1598 (12\%); Republik Karelien 283 (2,1\%); Gebiet von Kaliningrad 270 (2,0\%); Region von Sachalin 260 (1,95\%); Region von Chabarowsk 247 (1,85\%); Region von Krasnodahr 232 (1,7\%); Gebiet von Samara 220 (1,65\%); Gebiet von Murmansk 218 (1,6\%); Küstengebiet 171 (1,3\%); Gebiet von Wolgograd 171 (1,3\%); Region von Omsk 144 (1,1\%). In den 12 Gebieten wurden somit 9411 Unternehmen mit Auslandskapitaleinlagen angemeldet, 70,7\% der Gesamtzahl. Der größte Teil dieser Unternehmen arbeitet in Moskau und St. Petersburg, nämlich 7195 (oder 54\%). Die Hauptfaktoren dafür sind die Anerkennung dieser Städte als politische und finanzielle Zentren Rußlands und das deutlich höhere Niveau ihrer Infrastrukturentwicklung.

Zusammenfassend kann man sagen, daß die Zahl der Gemeinschafts- und Auslandsunternehmen und ihr Beitrag zur russischen Wirtschaft während der letzten fünf Jahre unablässig gestiegen ist. Dennoch ist das Wachstumstempo der Zahl dieser Betriebe wie auch des Ausmaßes der Auslandskapitaleinlagen und ihre Regional- und Bereichsstruktur unserer Meinung nach unbefriedigend. Beispielsweise wurden sogar im kleinen Estland Ende 1993 mehr als 14’000 
Joint Ventures und ausländische Unternehmen registriert. ${ }^{6}$ Auch nach Meinung des Abteilungsleiters für Politik- und Rechtsregulierung der Auslandskapitalanlagen im Wirtschaftsministerium, W. Lebedev, zeugt der Umfang des Auslandskapitals (Anfang 19954 Mrd. US\$) wie auch dessen jährlicher Zuwachs (1993 1,4 Mrd. US\$; 1994 1,05 Mrd. US\$; während der ersten 9 Monate 1995 - 1,57 Mrd. US\$) vom höchst bescheidenen Platz Rußlands in diesem Bereich.

Es gibt einige Gründe für diese Situation: Die wichtigsten Faktoren, die das Investitionsklima in jedem Land, auch in Rußland, beeinflussen, lassen sich in vier Gruppen unterteilen; die politische und die wirtschaftliche Situation eines Landes und das Niveau der gesetzlichen und infrastrukturellen Rahmenbedingungen, die Auslandskapital anziehen bzw. das Vorhandensein oder das Fehlen von Risiken, die diese Faktoren bedingen.

Auf dem Internationalen Kongreß „Das Gemeinschaftsgewerbe in Rußland“, der 1994 in St. Petersburg stattfand, wurde vermerkt, daß während der letzten Jahre in Rußland die Voraussetzungen für die Tätigkeit der Betriebe mit Auslandskapitaleinlagen im Vergleich mit den Bedingungen zur Zeit der Umgestaltung der ehemaligen UdSSR (Ende der 80er Jahre) wesentlich schlechter geworden sind. Während der Jahre 1992 und 1993 wurden durch neue wirtschaftliche Gesetzes und Erlasse die Vergünstigungen und Sicherheiten, die das „Gesetz über Auslandskapitaleinlagen“ enthält, aufgehoben oder wesentlich verändert. Es wurde die Zinsfreistellung, die zollfreie Ausfuhr selbst hergestellter Waren und die zollfreie Einfuhr von Gütern für den eigenen Produktionsbedarf abgeschafft; mit den höchsten Steuern sind die Werbungs-, Dienstreise- und Personalvorbereitungskosten belegt.

In der zweiten Hälfte 1993 (nach dem Präsidentenerlaß Nr. 1466 vom 27.09.1993 „Über eine Vervollkommnung der Arbeit mit Auslandsinvestitionen“, der allen Unternehmen mit Auslandskapitaleinlagen die Immunität gegen die Normativakte, die ihnen Materialverluste verursachen könnten, zusicherte) deutete sich wieder eine gewisse Wende hin zum in den meisten Ländern praktizierten Präferenzregime an. In der Folgezeit gab es noch einige weitere Erlasse des Präsidenten und der Regierung, die eine gewisse Liberalisierung der Auslandskapitalgebertätigkeit auf dem russischen Markt anstrebten Insbesondere wurden einige Ausfuhrtarifvorteile für die Sonderkategorie der Unternehmen mit Auslandskapitaleinlagen vorgesehen; es wurde die Befreiung von der Mehrwertsteuer und der Sondersteuer auf Ausrüstungen für den Produktionsbedarf verlängert; es wurden teilweise die Beschränkungen der Auslandsbankentätigkeit in Rußland aufgehoben usw.

6 O.V.: Die ausländischen Kapitaleinlagen in Rußland: Ob die neuen Maßnahmen die alten Probleme lösen werden. In: Die Wirtschaft und das Leben. Nr. 9. Bd. 30. 1995. (russ.) 
Zwecks weiterer Stimulierung ausländischer Investitionen in der russischen Wirtschaft wurde vom Präsidenten am 25.01.1995 der Erlaß Nr. 73 „Über die Zusatzmaßnahmen zum Anreiz von Auslandsinvestitionen für die produzierenden Bereiche“ herausgegeben. In diesem Erlaß wird die Senkung der Einfuhrzölle in Bezug auf einzelne Gruppen von Importgütern (außer Spirituosen und Tabakwaren) vorgesehen. Dabei werden die etablierten Vergünstigungen unter folgenden Bedingungen gewährt: vergünstigte Einfuhrzollraten sind nur anwendbar im Bezug auf Waren, deren Auslandslieferanten die Gründer von Betrieben sind, die ähnliche Produkte in Rußland unter Ausnutzung einheimischer Rohstoffe und Arbeiter herstellen und deren direkte Kapitaleinlagen in die Materialproduktionsbereiche Rußlands mindestens 100 Mio. US\$ betragen. Außerdem muß eine Kapitaleinlage des Auslandsteilnehmers mindestens 10 Mio. US\$ betragen.

Dieser Erlaß, wie auch verschiedene andere Präsidenten- und Regierungsakte zur Regulierung des rechtlichen Rahmens für Auslandsinvestitionen in Rußland, die während der letzten Jahren herausgegeben wurden, wird damit begründet, daß auf der einen Seite ein Anwachsen des Interesses westlicher Kapitalgeber für umfangreiche Investitionen auf dem russischen Markt festzustellen ist und auf der anderen Seite eine ganze Reihe von Negativfaktoren, diese Prozesse behindern.

Die wichtigsten Negativfaktoren muß man in der vorhandenen sozial-ökonomischen und politischen Instabilität und dem Ausmaß der politischen Risiken sehen. In der Zeitschrift „Economist“ wurde die Kennziffer des Wirtschaftsrisikos in Rußland mit 90\% angegeben (im Vergleich dazu bewegt sich die Kennziffer für andere ehemalige RGW-Länder Zentraleuropas zwischen 29\% und 40\%). Ein weiterer negativer Faktor ist die fortgesetzte Kapitalflucht aus Rußland, die in den Jahren 1993-1995 um ein vielfaches den Kapitalzufluß übertraf.

Unserer Meinung nach bedarf es für die Schaffung eines günstigen Investitionsklimas für Gemeinschafts- und Auslandsbetriebe in Rußland bestimmte gesetzliche und normative Neuerungen, in erster Linie:

- die Aufhebung der außertariflichen und tariflichen Beschränkungen der eigenen Produktionsausfuhr und der Rohstoff-, Fertigteile- und Materialeinfuhr zur eigenen Herstellungstätigkeit für Unternehmen mit ausländischen Kapitaleinlagen;

- Steuerbefreiung für einen Zeitraum von 3 bis 5 Jahren für Betriebe mit Auslandsinvestitionen in den Prioritätsbereichen;

- die Beseitigung der Besteuerung von Kapitalerhöhung und Gewinn von Unternehmen gemeinschaftlicher und ausländischer Eigentumsformen;

- wesentliche Verminderung der Gesamt-Steuerbelastung der Betriebe mit dem Ziel der Erhöhung ihres Investitionspotentials; 
- die Einführung von Konkursnormen für Unternehmen aller Eigentumsformen und Tätigkeitsrichtungen, unter der Bedingung der Schutzvorsorge für die Interessen der Kapitaleigentümer dieser Unternehmen.

Das breite System der wirtschaftlichen, rechtlichen, finanziellen, informatorischen und organisatorischen Maßnahmen für die Stimulierung von Auslandsinvestitionen wurde im „Komplexprogramm der Stimulierung der heimischen und ausländischen Kapitaleinlagen in die russische Wirtschaft“ realisiert, das von der Regierung Rußlands im Erlaß Nr. 1016 am 13.10.1995 gebilligt wurde. Alle diese Maßnahmen können zweifelsfrei nur beim Vorhandensein politischer und sozialer Stabilität im Lande, die eine sinnvolle Langfristplanung für russische und ausländische Unternehmer ermöglichen, auch eine Verbesserung des Investitionsklimas in Rußland bewirken. 